\title{
Low-frequency oscillations of the neural drive to the muscle are increased with experimental muscle pain
}

\author{
Dario Farina, ${ }^{1}$ Francesco Negro, ${ }^{1,4}$ Leonardo Gizzi, ${ }^{1,2}$ and Deborah Falla ${ }^{1,3}$ \\ ${ }^{1}$ Department of Neurorehabilitation Engineering, Bernstein Focus Neurotechnology Göttingen, Bernstein Center for \\ Computational Neuroscience, University Medical Center Göttingen, Georg-August University, Göttingen, Germany; \\ ${ }^{2}$ Dipartimento di Scienze del Movimento Umano e dello Sport, Università degli Studi di Roma "Foro Italico," Rome, Italy; \\ ${ }^{3}$ Pain Clinic, Center for Anesthesiology, Emergency and Intensive Care Medicine, University Hospital Göttingen, Göttingen, \\ Germany; and ${ }^{4}$ Department of Health Science and Technology, Aalborg University, Aalborg, Denmark
}

Submitted 5 April 2011; accepted in final form 29 October 2011

Farina D, Negro F, Gizzi L, Falla D. Low-frequency oscillations of the neural drive to the muscle are increased with experimental muscle pain. J Neurophysiol 107: 958-965, 2012. First published November 2, 2011; doi:10.1152/jn.00304.2011.-We investigated the influence of nociceptive stimulation on the accuracy of task execution and motor unit spike trains during low-force isometric contractions. Muscle pain was induced by infusion of hypertonic saline into the abductor digiti minimi muscle of 11 healthy men. Intramuscular EMG signals were recorded from the same muscle during four isometric contractions of 60 -s duration at $10 \%$ of the maximal force [maximal voluntary contraction (MVC)] performed before injection (baseline), after injection of isotonic (control) or hypertonic saline (pain), and 15 min after pain was no longer reported. Each contraction was preceded by three 3 -s ramp contractions from $0 \%$ to $10 \%$ MVC. The lowfrequency oscillations of motor unit spike trains were analyzed by the first principal component of the low-pass filtered spike trains [first common component (FCC)], which represents the effective neural drive to the muscle. Pain decreased the accuracy of task performance [coefficient of variation $(\mathrm{CoV})$ for force: baseline, $2.8 \pm 1.8 \%$, pain, $3.9 \pm 1.8 \% ; P<0.05]$ and reduced motor unit discharge rates $[11.6 \pm$ 2.3 pulses per second (pps) vs. $10.7 \pm 1.7 \mathrm{pps} ; P<0.05]$. Motor unit recruitment thresholds $(2.2 \pm 1.2 \%$ MVC vs. $2.4 \pm 1.6 \% \mathrm{MVC})$, interspike interval variability $(18.4 \pm 4.9 \%$ vs. $19.1 \pm 5.4 \%)$, strength of motor unit short-term synchronization [common input strength (CIS) $1.02 \pm 0.44$ vs. $0.83 \pm 0.22$ ], and strength of common drive $(0.47 \pm 0.08$ vs. $0.47 \pm 0.06)$ did not change across conditions. The FCC signal was correlated with force $(R=0.45 \pm 0.06)$, and the $\mathrm{CoV}$ for FCC increased in the painful condition $(5.69 \pm 1.29 \%$ vs. $7.83 \pm$ $2.61 \% ; P<0.05)$. These results indicate that nociceptive stimulation increased the low-frequency variability in synaptic input to motoneurons.

motor unit; common principal component; interspike interval; shortterm synchronization; common drive

ACCURACY IN THE GENERATION of force depends on several neuromuscular mechanisms (Marmon et al. 2011), including the variability of synaptic input received by the motoneurons, which is reflected in the neural drive to muscles. The lowfrequency bandwidth of the motoneuron output spike trains has the most profound influence on the accuracy of force generation during static contractions, because of the low-pass filtering effect of the transformation of the neural drive to muscles into force (Negro et al. 2009). The characteristics of the low-

Address for reprint requests and other correspondence: D. Farina, Dept. of Neurorehabilitation Engineering, Bernstein Center for Computational Neuroscience, Univ. Medical Center Göttingen, Georg-August Univ., Von-Siebold-Str. 4, 37075 Göttingen, Germany (e-mail: dario.farina@bccn.uni-goettingen.de). frequency components of motor unit spike trains are determined by the integration of supraspinal and afferent synaptic input to the motoneurons. When the afferent input is modified, for example, because of nociceptive stimulation, the central nervous system needs to compensate for this alteration by modulating the descending drive in order to maintain motor performance with minimal change.

Nociceptive stimulation influences muscle control, as has been assessed in several experimental pain studies (for a recent review, see Hodges and Tucker 2011). For example, painful injection of hypertonic saline (Graven-Nielsen et al. 1997a) decreases the maximal force generation capacity of a muscle (Graven-Nielsen et al. 1997b). Moreover, during static contractions, pain decreases the discharge rate of the active motor units (Farina et al. 2004, 2005) by activation of small-diameter muscle afferents that have projections to the motoneurons and influence the descending drive (Gandevia 2001). These changes may be associated with a decrease in performance when executing a task. However, contractions that do not pose high requirements on the system can be executed with an almost unchanged motor output, because of the redundancy of the motor system. For example, submaximal force levels can be reached in painful conditions similar to nonpainful tasks (see, e.g., Farina et al. 2004).

Although simple tasks can be executed without changes in motor output in painful conditions, the motor performance in more complex tasks is influenced by pain. For example, the accuracy of task execution may be influenced by pain, depending on the requirements on the system. Indeed, although force steadiness for leg muscles (Farina et al. 2004) and dexterity for a precision grip task of hand muscles (Smith et al. 2006) do not change with muscle pain, the neural control of muscles may worsen with pain during tasks that require fine control (Bandholm et al. 2008; Christou et al. 2004). These results are also consistent with observations in patients with chronic pain (see, e.g., Muceli et al. 2011).

In this study we analyzed accuracy in the execution of abduction of the fifth digit under conditions of experimental muscle pain. Because this is an unaccustomed task not often performed, we hypothesized that pain would influence its accuracy, assessed as force steadiness. After verifying this hypothesis, we analyzed the oscillations of one common lowfrequency component in motor unit spike trains, which has been shown to characterize the effective neural drive to muscles for force production (Negro et al. 2009). The aim of the 
study was to identify the neural determinants of impaired control of an accurate task with nociceptive stimulation.

\section{MATERIALS AND METHODS}

Subjects. Eleven healthy men [age $25.1 \pm 2.5 \mathrm{yr}$ (mean $\pm \mathrm{SD}$ )] participated in the study. The study was conducted in accordance with the Declaration of Helsinki and approved by the local ethics committee (N-20090019). The subjects provided written informed consent before participation.

EMG recordings. Single motor unit action potentials were recorded from the right abductor digiti minimi muscle with intramuscular EMG. Intramuscular EMG was measured with Teflon-coated stainless steel wires (diameter $0.1 \mathrm{~mm}$; A-M Systems, Carlsborg, WA) inserted with one 25-gauge hypodermic needle. One pair of wires was placed in the proximal portion of the muscle belly (Negro et al. 2009). The needle was removed after insertion, leaving the wires inside the muscle. Each wire was cut to expose the cross section of the tip without insulation. The bipolar intramuscular EMG signal was amplified (Counterpoint EMG, Dantec Medical, Skovlunde, Denmark), band-pass filtered $(500 \mathrm{~Hz}$ to $5 \mathrm{kHz})$, and sampled at $10 \mathrm{kHz}$. A reference electrode was placed around the right wrist.

The position of the wires was slightly adjusted before the recordings started, and when the signal quality was poor, which occurred rarely, a new pair of wires was inserted.

Experimental muscle pain. Experimental muscle pain was induced by injection (27-gauge cannula) of $0.5 \mathrm{ml}$ of sterile hypertonic saline $(5.8 \%)$ into the right abductor digiti minimi muscle. Isotonic saline $(0.5 \mathrm{ml}, 0.9 \%)$ was used as a control injection at the same location. The location of the injection was $\sim 10 \mathrm{~mm}$ distal and $\sim 5 \mathrm{~mm}$ transverse to the intramuscular wires. The bolus was manually injected over a 10 -s period.

Participants performed isometric fifth finger abduction under four conditions: baseline, isotonic saline, hypertonic saline, and postpain. The conditions of baseline and isotonic saline were randomized across subjects and were always followed by hypertonic saline and postpain conditions. Thus for each subject the isotonic saline injection was given before the hypertonic injection; however, subjects were advised that both injections may or may not be painful. A rest of 5 min was given between the first three conditions. The set of contractions in the postpain condition was performed 15 min after pain was no longer reported by the subject.

The participants were asked to verbally rate their level of perceived pain intensity on an 11-point numerical rating scale (NRS) anchored with "no pain" and "the worst possible pain imaginable." Pain intensity ratings were obtained immediately after the injections and every $30 \mathrm{~s}$ until pain was no longer reported.

Procedure. The subject was seated on an adjustable chair with the right arm extended in a force brace (Aalborg University). The fifth finger was fixed in the isometric device for measurement of finger abduction forces (Fig. 1). The forearm and the first four digits were secured with Velcro straps. The force produced by the fifth finger was measured with two force transducers (Interface), one in the transverse plane (abduction force) and the other in the sagittal plane (flexion force). The force signal was sampled at $10 \mathrm{kHz}$ and stored on a computer. Visual feedback on finger abduction force was provided on an oscilloscope.

The subjects performed three maximal voluntary contractions (MVCs) of fifth finger abduction with a rest of $2 \mathrm{~min}$ in between. The maximum force achieved during the maximal contractions was considered as the reference MVC. Five minutes after the MVCs, the subject performed three ramp contractions, separated by $10 \mathrm{~s}$, with force increasing from $0 \%$ to $10 \% \mathrm{MVC}$ in $3 \mathrm{~s}$. After the ramp contractions, the subject further performed an isometric contraction at $10 \% \mathrm{MVC}$ for $60 \mathrm{~s}$. The same set of contractions (ramp and sustained) was repeated for the isotonic, hypertonic, and recovery conditions. The subject received feedback on force during all contractions and was asked to maintain the target force level as accurately as possible. The target force output was displayed on a PC monitor located $1 \mathrm{~m}$ in front of the subject with the gain adjusted to view $\pm 10 \%$ of the target force value on a 20 -cm-high display. Specifically during the static contractions, subjects were requested to match the actual force output as closely as possible to the target force for the full duration of the trial.

In 5 of the 11 subjects, we repeated the same experimental procedure on a different day, but we injected hypertonic $(0.5 \mathrm{ml}, 5.8 \%)$ or isotonic saline $(0.5 \mathrm{ml}, 0.9 \%)$ into the belly of the tibialis anterior muscle instead of the abductor digiti minimi muscle. In these tests, EMG signals were not recorded and force steadiness was evaluated for contractions of the abductor digiti minimi muscle with the same procedures as in the main experimental session. These additional tests served to exclude the possibility that force steadiness was influenced indirectly by the painful stimulus because it may have diverted attention away from the task (attention control experiment).

Signal and data analysis. The action potentials of individual motor units were identified from the intramuscular EMG signals by the use of a decomposition algorithm (McGill et al. 2005). This interactive algorithm includes a user interface for manually editing and verifying the results. The software displays a segment of the EMG signal, the

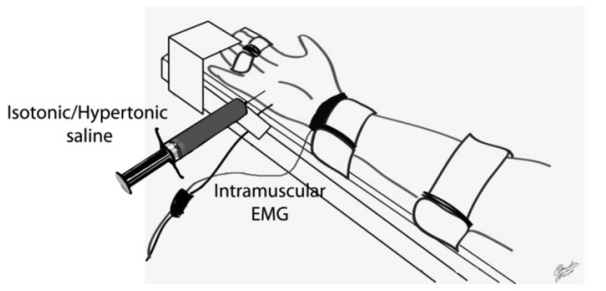

RECORDING SET
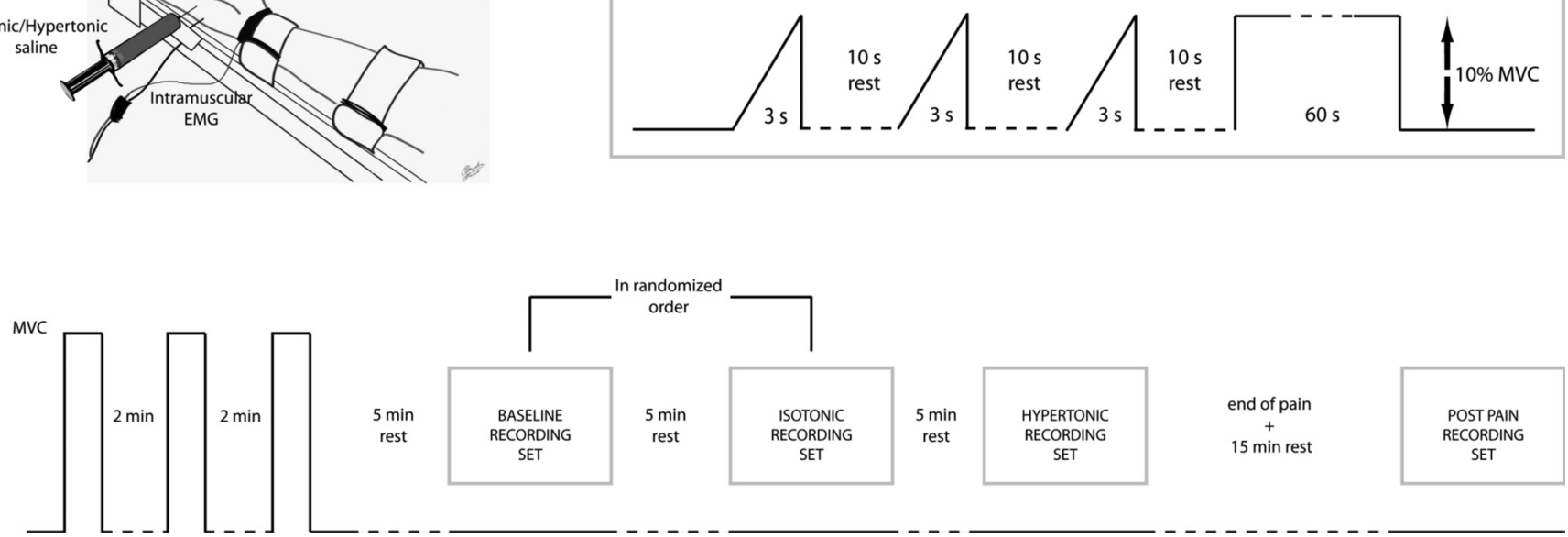

Fig. 1. Experimental setup and recording sequence. The baseline and isotonic saline recordings (randomized) were followed by the hypertonic saline and postpain recordings. Each recording set included ramp and static contractions. MVC, maximal voluntary contraction. 
templates of the action potentials of the identified motor units, the discharge patterns, and a close-up of the signal for resolving missed discharges and superimpositions. Accuracy of the automatic decomposition was achieved by inspection of the identified discharge patterns. Full, regular patterns provided confidence that the decomposition was correct, whereas gaps, extra discharges, or uneven intervals provided an indication of possible decomposition errors. To assist in identifying missed discharges the program displays bars in the signal panel that indicate the expected discharge times of each motor unit. The signal portion can then be viewed in the close-up panel, which displays the signal at an expanded scale and allows matching motor unit templates to be selected. The close-up panel also displays superimpositions at an expanded scale that allows verification of the result or allows different sets of templates to be selected and adjusted to find the correct fit. Each motor unit spike train was manually edited by an experienced operator, and, specifically, any unusually long ( $>250 \mathrm{~ms}$ ) or short $(<20 \mathrm{~ms})$ interspike intervals (ISIs) were manually inspected to observe for potential discrimination errors. Previous extensive validation of this decomposition algorithm proved an accuracy of $>98 \%$ in conditions similar to those of the present study (McGill et al. 2004). Although the algorithm allows manual identification of potential decomposition errors, the action potentials are always automatically inserted in the time instants that minimize the mean square error, so that there are no arbitrary choices by the operator on the time of occurrence of the action potentials. The temporal resolution in the identification of action potentials is very high since the signals are resampled to $100 \mathrm{kHz}$ for decomposition. Figure 2 shows an example of identification of action potentials from the superposition of five action potential occurring almost at the same time instant. The timing for each action potential is in this case estimated by the algorithm with an error below the time resolution $(0.01 \mathrm{~ms})$ and thus negligible. Estimation of the exact timing is critical for assessing short-term synchronization. The validation of the decomposition method in identifying the action potentials and their times of occurrence with high accuracy provided by McGill et al. (2004) as well as a number of tests we performed in this (representative example in Fig. 2) and previous studies (e.g., Negro et al. 2009) indicate that the method used for decomposition in this study is sufficiently accurate to provide precise information on motor behavior.

The analysis of the decomposed spike trains was performed on the motor units that could be identified in all four conditions for each subject. The identification of the same units was based on the shape of their action potentials, with a cross-correlation criterion on shape specified in RESULTS. The following analyses were applied to motor units recorded in all conditions, unless stated otherwise.

The recruitment threshold of each motor unit was measured from the ramp contractions as the force corresponding to the first discharge separated by the next of $<250 \mathrm{~ms}$. This force value was averaged across the three ramp contractions. The instantaneous discharge rate of each motor unit was smoothed with a Hann window of duration 400 $\mathrm{ms}$ and high-pass filtered with a cutoff frequency of $0.75 \mathrm{~Hz}$ [zerophase filter, $H(f)=1-\sin (\pi f) / \pi f]$ to remove offsets and trends, as proposed in previous studies (De Luca et al. 1982). The resulting smoothed and detrended discharge rates (referred to below as smoothed discharge rates, for simplicity) were arranged in a matrix (time samples $\times$ motor unit), and their principal components were computed with the eigenvalue decomposition of their covariance matrix, as proposed by Negro et al. (2009). The maximum eigenvalue of the covariance matrix, which corresponds to the relative power of the first principal component, was used to quantify the strength of the common low-frequency oscillations in the activities of the identified motor units (Negro et al. 2009). It has been shown that this is a measure of common drive that is consistent with other definitions (De Luca et al. 1982) but has greater sensitivity. Moreover, we analyzed the characteristics of the first principal component signal. The first principal component is a signal that projects the largest common variations in the smoothed motor unit discharge rates. This signal will be referred to as the "first common component" (FCC) of the lowfrequency oscillations of motor unit discharge rates, as described by Negro et al. (2009). The mean frequency of oscillation of the FCC was computed as the first-order moment of the power spectrum of the FCC signal.

The degree of motor unit short-term synchronization was calculated with cross-correlation histograms (bin width $1 \mathrm{~ms}$ ) between $-100 \mathrm{~ms}$ prior to and $100 \mathrm{~ms}$ after the discharge of the reference unit (Nordstrom et al. 1992). The cumulative sum (CUSUM) technique was used to assess the location of the peak of the histograms and the peak width, as described previously (Davey et al. 1986; Ellaway et al. 1978; Semmler et al. 1997). The strength of short-term synchronization was measured as the common input strength (CIS) index, defined by Nordstrom et al. (1992). CIS values reported are the average over all pairs of motor units identified for each contraction (Semmler and Nordstrom 1999).

It has been suggested that ISI variability may also influence force steadiness (Enoka et al. 2003). Therefore, the coefficient of variation $(\mathrm{CoV})$ for the ISI and the force signal was computed as the ratio (\%) between the SD and the mean values, after removal of the linear trends. The $\mathrm{CoV}$ for the FCC signal was computed in a similar way, as the ratio between the SD of the detrended FCC signal and its mean value. For all variables, the $\mathrm{CoV}$ was computed with intervals of 5-s duration and the values obtained were averaged over the contraction duration.

Statistical analysis. The pain scores and the variables that characterized the motor unit spike trains were analyzed with ANOVA followed by pairwise comparisons with Student-Newman-Keuls (SNK) post hoc tests. For some analyses the factors were condition (baseline, isotonic saline, hypertonic saline, post) and time (beginning or end of contractions) (2-way ANOVA), whereas in other cases the factor was condition (2-way ANOVA), as specified in RESULTS. Linear correlation analyses were performed by computing the Pearson correlation coefficient. Results are presented as means and SD. The significance level was set to $P<0.05$.

\section{RESULTS}

Injection of hypertonic saline into the abductor digiti minimi muscle elicited a painful sensation that lasted for the entire set of ramp and sustained contractions. The NRS score for pain was $4.1 \pm 1.8$ at the beginning of the painful set of contractions and decreased, although not significantly, to $3.7 \pm 2.2$ by the end of the set of contractions. The NRS score during the contractions following the injection of isotonic saline was $0.8 \pm 0.9$ (average across the beginning and end of the contraction), significantly lower than with the hypertonic saline injection $(P<0.0001)$.

A total of 35 motor units could be identified as active in all contractions (ramp and sustained contractions in the 4 experimental conditions) $(3 \pm 1$ per subject). For these motor units, the correlation coefficient between action potential shapes in the different conditions was $>0.9$, indicating the stability of the recordings. Figure 3 presents raw experimental signals for the four conditions and the motor units identified as common to all conditions in a representative subject. The motor units that were investigated and that were thus active in all conditions did not change their recruitment thresholds across the four conditions (Table 1), as estimated from the ramp contractions.

In the representative example of Fig. 3, the force variability increased after injection of hypertonic saline $(\mathrm{CoV}$ for force, $4.1 \%$ ) with respect to the other conditions (average over the other conditions, 3.2\%). This observation also applied to the group data, for which the $\mathrm{CoV}$ for force was significantly greater in the painful condition with respect to the other 


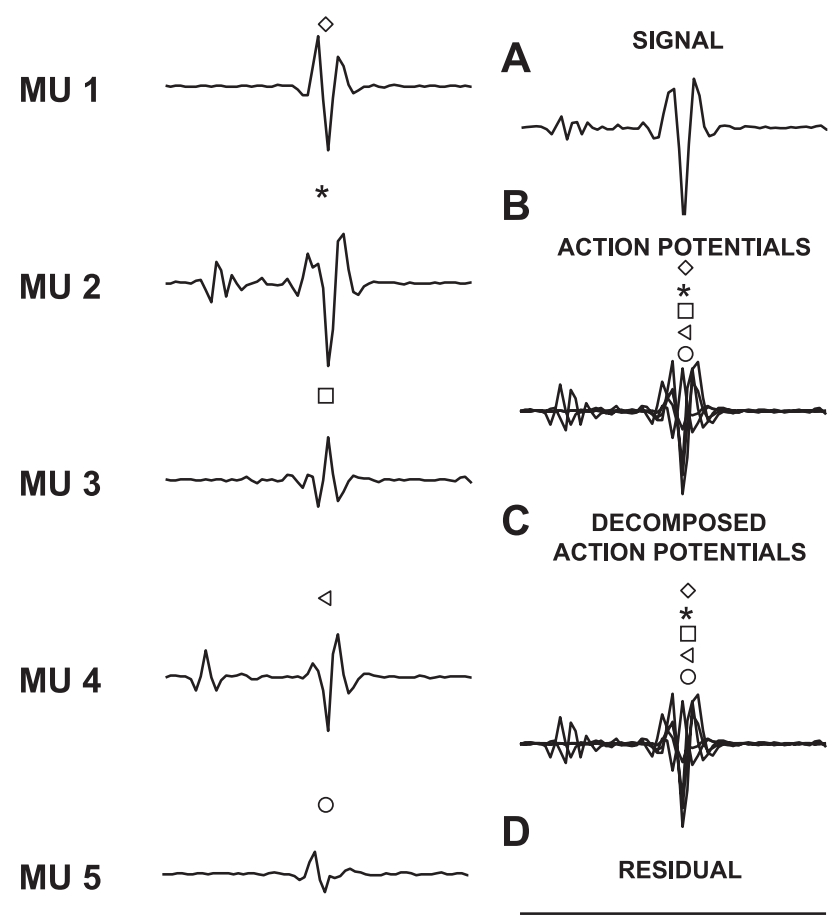

Fig. 2. An example of superimposition of 5 action potentials and their identification by the algorithm used for EMG decomposition. The action potentials are experimental potentials of 5 motor units (MUs; shown on left) that have been located at specific time instants and summed to generate a synthetic superposition. $A$ : the synthetic signal. $B$ : the location in time of the 5 action potentials in the synthetic signal. $C$ : the location in time of the 5 action potentials as determined by the decomposition algorithm applied to the signal in $A$. $D$ : the signal has been reconstructed by adding the action potentials as identified by the decomposition algorithm (at the time instants estimated by the decomposition algorithm) and subtracted from the original signal to determine the residual. Symbols in $B$ and $C$ indicate the position of the 5 action potentials (almost fully overlapping). In this example, the decomposition algorithm correctly identified all action potentials and the time instants of their occurrence (with error on the time instants smaller than the time resolution of $0.01 \mathrm{~ms}$ ).

conditions (ANOVA: $F=5.6, P<0.01$; SNK: $P<0.05$; Table 1). However, the $\mathrm{CoV}$ for force did not change over time and was not statistically different between the beginning and the end of the contraction (Table 1). Therefore, the subjects could maintain the target force level in all conditions but with decreased steadiness in the painful condition.

The average motor unit discharge rate was lower during the painful condition than in the other conditions (ANOVA: $F=$ 2.9, $P<0.05$; SNK, $P<0.05)$ and decreased from the beginning to the end of the contraction (ANOVA: $F=15.7$, $P<0.01$; SNK, $P<0.01$ ) (Table 1). The CoV for ISI was not different across conditions and did not change significantly between the beginning and the end of the contraction (average across conditions and time intervals, $20.2 \pm 5.1 \%$; Table 1). Moreover, there was no association between the $\mathrm{CoV}$ for force and the CoV for ISI $\left(R^{2}<0.18\right.$ across subjects for all conditions at the beginning and the end of the contraction; all $P>0.05)$. Therefore, the variability in ISI did not influence the variability in force and did not determine its increase during the painful contractions.

Figure 4 shows the cross-correlation function and crosshistogram between the spike trains of a pair of motor units in the four conditions for one subject. The cross-correlation function measures the strength of common drive, whereas the cross-histogram reveals the strength of short-term synchronization. Both represent the degree of correlation among motor unit spike trains, although in different frequency bands (Negro and Farina 2011b). In this representative example, the strengths of common drive and of short-term synchronization were similar across conditions. For the group data, the number of motor unit pairs analyzed was 40 . The average strength of common drive did not vary across conditions (average across conditions $0.49 \pm 0.10$; Table 1) and was consistent with values reported in previous work (Negro et al. 2009). The CIS value as well as the width of the peak of the cross-histogram were also similar across conditions (averages $0.92 \pm 0.32$ and $13.4 \pm 5.8 \mathrm{~ms}$, respectively; Table 1) and consistent with earlier studies (Dideriksen et al. 2009; Negro et al. 2009). It was therefore excluded that altered strength of common drive or synchronization determined the increase in force variability with pain.

The low-frequency oscillations of motor unit spike trains were investigated by extracting the FCC signal (Negro et al. 2009). This signal was previously shown to be highly corre-

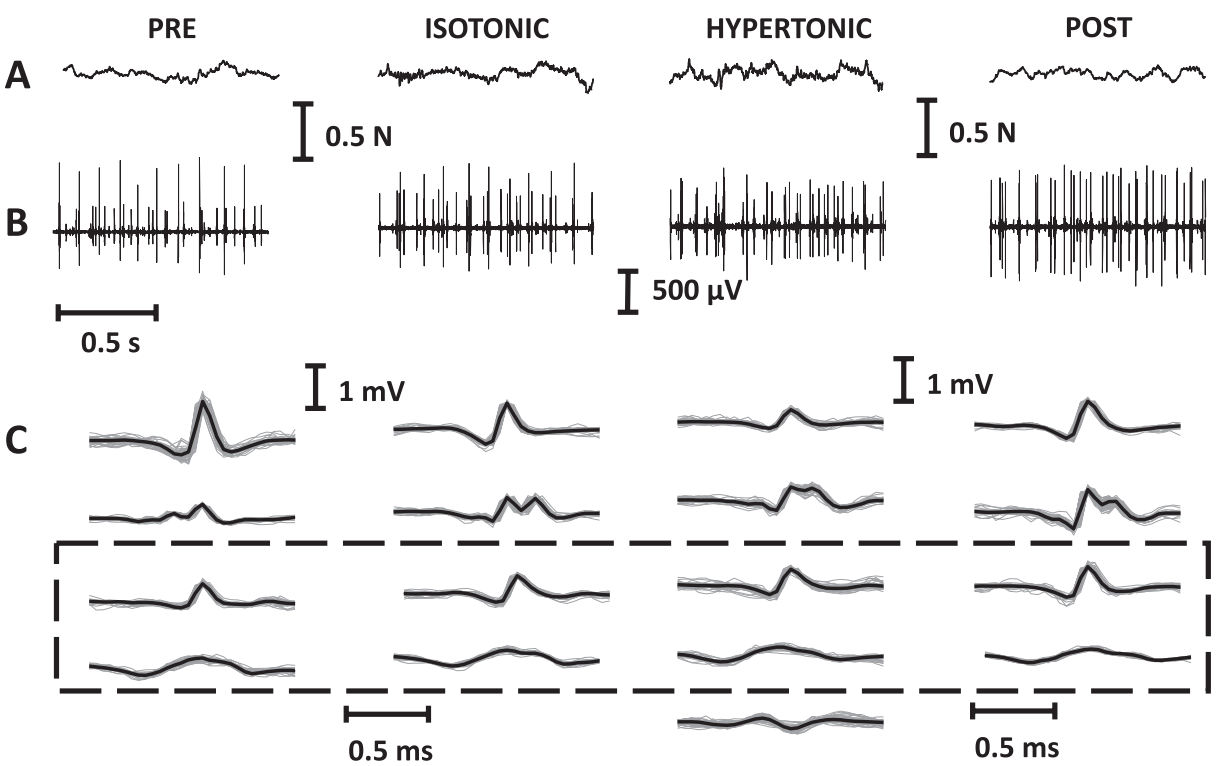

Fig. 3. Example of recorded data from 1 subject. $A$ : force signals recorded in the 4 conditions. $B$ : intramuscular EMG. $C$ : average templates of the decomposed motor units. The dashed box indicates the 2 motor units that have been identified as active in the 4 conditions (cross-correlation coefficient between their action potentials > $0.9)$, whereas the motor units outside the dashed box are those that could not be identified in at least 1 of the conditions (and were thus not included in the main analysis). 


\begin{tabular}{|c|c|c|c|c|}
\hline & \multicolumn{4}{|c|}{ Condition } \\
\hline \multicolumn{5}{|l|}{ Mean discharge rate, pps } \\
\hline Beginning of contraction (10 s) & $11.6 \pm 2.3$ & $10.7 \pm 1.7^{*}$ & $11.4 \pm 1.7$ & $12.1 \pm 2.0$ \\
\hline \multicolumn{5}{|l|}{ Mean discharge rate, pps } \\
\hline \multicolumn{5}{|l|}{ End of contraction $(10 \mathrm{~s})$} \\
\hline \multirow{2}{*}{\multicolumn{5}{|c|}{$\mathrm{CoV}$ for force, $\%$}} \\
\hline & & & & \\
\hline End of contraction $(10 \mathrm{~s})$ & $2.9 \pm 1.9$ & $3.8 \pm 1.8^{*}$ & $2.5 \pm 1.0$ & $2.7 \pm 1.4$ \\
\hline \multicolumn{5}{|l|}{$\mathrm{CoV}$ for ISI, $\%$} \\
\hline Beginning of contraction (10 s) & $18.4 \pm 4.9$ & $19.1 \pm 5.4$ & $18.7 \pm 5.6$ & $20.4 \pm 5.9$ \\
\hline \multicolumn{5}{|l|}{$\mathrm{CoV}$ for ISI, $\%$} \\
\hline End of contraction $(10 \mathrm{~s})$ & $18.0 \pm 5.9$ & $19.6 \pm 6.3$ & $18.5 \pm 6.9$ & $20.7 \pm 7.5$ \\
\hline Common drive index & $0.47 \pm 0.08$ & $0.47 \pm 0.06$ & $0.51 \pm 0.09$ & $0.51 \pm 0.10$ \\
\hline Beginning of contraction (10 s) & $5.69 \pm 1.29$ & $7.83 \pm 2.61 *$ & $5.76 \pm 1.65$ & $5.92 \pm 1.47$ \\
\hline \multicolumn{5}{|l|}{$\mathrm{CoV}$ for FCC, \% } \\
\hline End of contraction $(10 \mathrm{~s})$ & $6.15 \pm 2.39$ & $6.87 \pm 2.29^{*}$ & $6.05 \pm 2.54$ & $5.49 \pm 2.03$ \\
\hline
\end{tabular}

Values are group means \pm SD. FCC, first common component; ISI, interspike interval; CoV, coefficient of variation; pps, pulses per second; MVC, maximal voluntary contraction; CIS, common input strength. *Significantly different with respect to the other conditions $(P<0.05)$.

lated to the force signal in normal conditions since it can be considered as the effective neural drive transmitted to the muscle, after the low-pass filtering effect of the twitch forces (Negro et al. 2009). As in previous work (Negro et al. 2009), in this study the FCC signal was significantly correlated to the force signal (peak of the cross-correlation function between the 2 signals significantly different from 0 ; average values in Table 1). The strength of this correlation did not depend on the
A

PRE

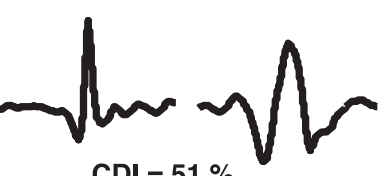

B

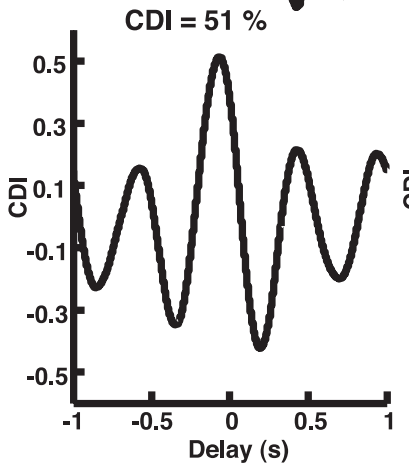

C

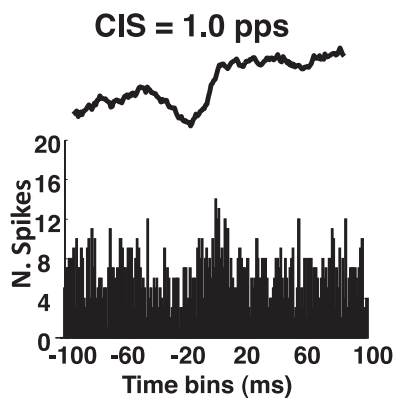

ISOTONIC

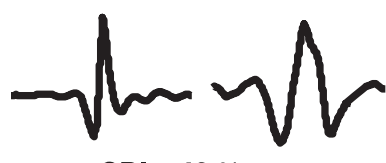

$\mathrm{CDI}=\mathbf{4 9} \%$
HYPERTONIC

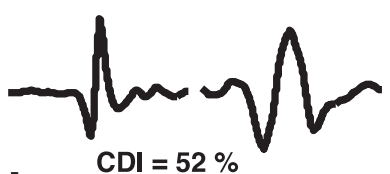

POST

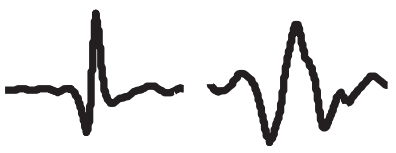

CDI $=50 \%$

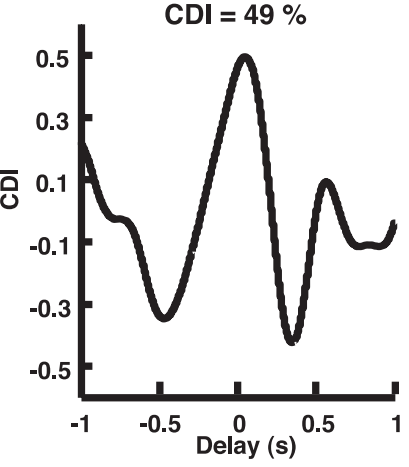

$\mathrm{CIS}=0.7 \mathrm{pps}$

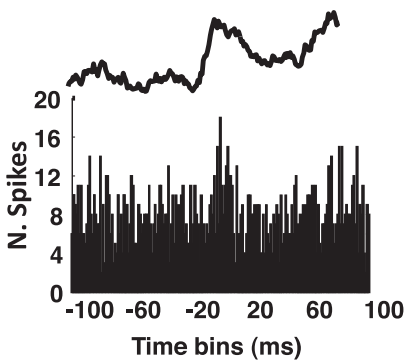

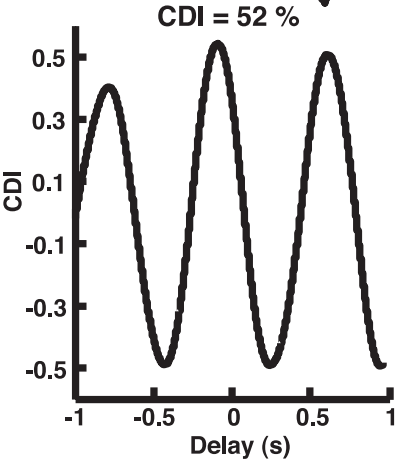
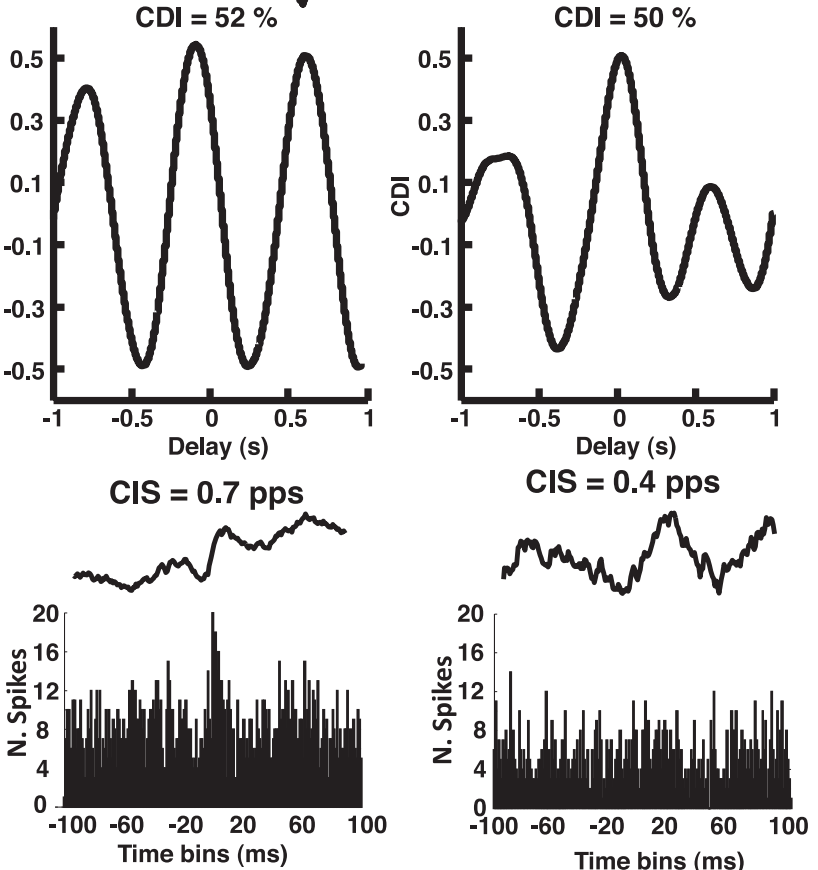

Fig. 4. Common drive and synchronization for motor units active in the 4 conditions in a representative subject. $A$ : action potentials of 2 motor units identified in the 4 contractions. $B$ : cross-correlation between the average discharge rates of the 2 motor units across the various conditions. $C$ : frequency histograms and cumulative sum (CUSUM) plots estimated from the motor units in A across conditions. CDI, common drive index; CIS, common input strength; pps, pulses per second. 
condition (average over the 4 conditions, $R=0.45 \pm 0.06$ ), which indicates that the FCC described well the force oscillations in all conditions. Figure 5 shows the FCC and the force signal in the four conditions for a representative subject. In this example, the variability of the FCC signal is greater in the painful condition than in the other conditions. For the group data, the variability of the FCC was quantified with the CoV for the FCC and was found to be significantly greater in the painful condition than in the other conditions (ANOVA: $F=$ $3.5, P<0.05$; SNK: $P<0.05)$, although it did not depend on time (Table 1). In agreement with the correlation between FCC and force signal, the CoV for these two signals were positively correlated across subjects (average across the 4 conditions and the 2 time intervals, $R^{2}=0.31 \pm 0.10$; all $P<0.05$ ). Therefore, the increased force variability was associated with increased amplitude of the common low-frequency components in motor unit spike trains. The mean frequency of the FCC signal did not depend on the condition (average $1.8 \pm 0.1 \mathrm{~Hz}$ ).

The above results were all obtained from the analysis of those motor units that were identified in all four conditions, as indicated above. The same analyses were also repeated for the entire population of identified motor units in each condition, i.e., without restriction to the motor units identified in all conditions. The total number of motor units for this analysis was 205. All the results reported above were confirmed for this larger group of motor units. In summary, the average discharge rate was lower in the painful condition $[n=205,10.6 \pm 1.6$ pulses per second (pps)] than at baseline (11.4 $\pm 2.3 \mathrm{pps} ; P<$ 0.05 ). Moreover, the ISI variability (baseline: $20.1 \pm 5.3 \%$, pain: $19.8 \pm 4.9 \%)$ and strength of common drive $(0.52 \pm 0.12$ vs. $0.48 \pm 0.08)$ and of short-term synchronization (1.01 \pm 0.40 vs. $0.98 \pm 0.35)$ did not change across conditions, whereas the variability of FCC increased in the painful condition $(6.1 \pm 2.3 \%$ vs. $7.6 \pm 1.9 \% ; P<0.05)$.

Finally, injection of hypertonic saline into the tibialis anterior muscle in the attention control experiment ( $n=5$ subjects) elicited a painful sensation in the tibialis anterior with a NRS score similar to that for the injection in the abductor digiti minimi muscle (beginning of the set of contractions: $3.9 \pm 2.1$; end: $3.8 \pm 2.4$ ), whereas the NRS score during the contractions following the injection of isotonic saline was very low $(0.3 \pm$ $0.1 ; P<0.0001$ with respect to hypertonic injection). In the attention control experiment, $\mathrm{CoV}$ for force during contractions of the abductor digiti minimi muscle did not significantly change with condition (baseline: $2.9 \pm 1.5 \%$, hypertonic saline

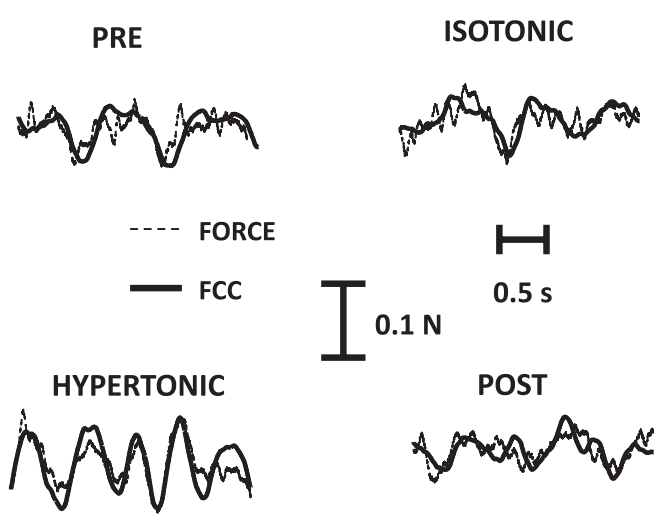

Fig. 5. First common component (FCC) and force. Comparison between the estimated FCC and the force signal in 1 subject during the 4 conditions. in the tibialis anterior muscle: $2.8 \pm 1.7 \% ; P>0.05$ ). This result excluded the possibility that pain by itself caused decreased task accuracy because of decreased attention to the task.

\section{DISCUSSION}

Nociceptive stimulation influenced the variability in the effective neural drive to the muscle (FCC) and, as a consequence, the accuracy in execution of a precise unaccustomed task. Conversely, the ISI variability, the strength of common drive, and the degree of short-term synchronization were not influenced by pain. Finally, pain did not influence the recruitment thresholds of the motor units that were active in the painful and nonpainful conditions.

The results on motor unit discharge rates confirmed those of previous work showing decreased motor unit discharge rates following experimentally induced muscle pain (Farina et al. 2004, 2005) and during sustained contractions (Gandevia 2001). Recent studies also indicate that this effect may not be uniform for all motor units and that different populations of motor units are likely recruited in painful conditions with respect to baseline (Tucker et al. 2009). In the present study, we chose to focus the main results on the motor units that could be identified in all four conditions, based on the shape of their action potentials. There were several action potential shapes that could be identified in only some of the conditions (e.g., Fig. 3). However, it is not possible to infer from the present data whether the recruitment order was altered because of pain, since small differences in force direction or small displacements of the recording wires may determine changes in the number of recruited units and in the shape of their action potentials. Nonetheless, the motor units that could be classified as common in all conditions did not change their recruitment threshold, as analyzed from the ramp contractions (Table 1). Moreover, when all the identified motor units were analyzed (including those not common to all conditions), the average discharge rates decreased significantly in the painful condition, as was observed for the common units. These results indicate that the majority of the motor units exhibited a decrease in discharge rate due to pain. The maintenance of the average force despite a decrease in motor unit discharge rates has been discussed in previous work (Farina et al. 2004, 2008; Hodges et al. 2008), without a definitive answer. The mechanism of a differential effect of pain on different motor units identified by Tucker et al. (2009) may partly explain the maintenance of force when the average discharge rate decreases, since different motor units may contribute differently to the total force.

The main contribution of this study is the analysis of the neural determinants of impaired accuracy in force maintenance with nociceptive input. Some previous studies reported unchanged variability in force during painful static contractions with respect to control conditions (e.g., Farina et al. 2004), while others reported an increase in force variability (Bandholm et al. 2008; Christou et al. 2004). The results of the present study indicate that for precise tasks, the demands of control cannot be met in painful conditions and thus the motor output is impaired. This is consistent with clinical observations in patients with chronic pain (Muceli et al. 2011). The main- 
tenance of a stable force level is determined by the neural drive that the muscle receives, which is generated by the combination of the descending drive from supraspinal centers and the afferent input, integrated and transmitted through the spinal motoneurons.

To characterize the effective neural drive to the muscle, we investigated the signal extracted from the common lowfrequency components of the motor unit spike trains by principal component analysis, a method that we recently proposed (Negro et al. 2009). Because the force output can be modeled as a low-pass filtered version of the cumulative motoneuron spike trains, the low-frequency components of the neural signal (FCC) are effective in determining the characteristics of the generated force (Fig. 5). Conversely, the high-frequency components are poorly transmitted to the force signal. Similarly, noncommon components are also filtered in the process of force production because of the summation of the forces exerted by the ensemble of active motor units. The extraction of FCC can be performed from a small number of detected motor units, because of the largely spread synaptic input to the motoneurons (Negro et al. 2009; Negro and Farina 2011a).

The FCC signal correlated with force, and its variability increased in the painful conditions, in association with an increase in force variability. As the low-frequency components in the output spike trains of motoneurons are related to the synaptic input received by the motoneurons, this result indicates an increased variability in synaptic input (lowfrequency band). The variability of FCC is related to the ability of the central nervous system to generate a descending drive that, combined with afferent input, produces a stable neural drive to muscles. Thus the increase in variability in this signal indicates an impairment of the central nervous system's ability to accurately control force in the investigated conditions of nociceptive input. This may be due either to the inability to fully compensate with the descending drive for an altered afferent input during nociceptive stimulation or to an effect of nociceptive input on the generation of the descending drive. The present data do not allow these two potential effects to be separated since they are derived from the motoneuron output. However, it is known that pain centrally influences other aspects of the motor output, such as the maximal force generation capacity (Graven-Nielsen et al. 2002); thus an increased variability in descending drive due to nociceptive input may be hypothesized. Finally, it is worth noting that, as reported previously (Negro et al. 2009), the FCC explained only a part of the variance of the force signal. Therefore, a characterization of the force signal by FCC still lacks some important components that may characterize force oscillations.

Since force steadiness may be influenced by several characteristics of the neural drive to the muscle, in this study we also investigated other potential sources of variability in addition to the low-frequency bandwidth of the cumulative spike train. ISI variability has been often associated with force variability (Enoka et al. 2003). ISI variability is due to the synaptic noise and its interaction with the time course of the postspike after hyperpolarization phase of the motoneuron (Matthews 1996). An increase in inhibitory synaptic input to the motoneurons, as occurs with nociceptive stimulation, may be associated with an increase in excitatory inputs, such as an increase in descending drive, so that the discharge rates are maintained almost unchanged and force does not decrease. This balanced increase in inhibitory and excitatory inputs may increase the synaptic noise (Berg et al. 2007). However, in this study ISI variability was similar across conditions, presumably indicating an unchanged level of synaptic noise. It must be noted that the ISI variability is mainly reflected in the high-frequency components of the motor unit spike trains, which would be poorly transmitted to force because of low-pass filtering by the motor unit twitches. Indeed, ISI variability did not correlate with the $\mathrm{CoV}$ for force in any conditions.

Steadiness in force may also be influenced by the degree of short-term synchronization (Yao et al. 2000). However, direct analysis of cross-histograms of pairs of motor unit spike trains indicated that the degree of synchronization was not influenced by pain (Fig. 4). Similarly, the strength of common drive, which represents the strength of common input to motoneurons in another frequency band with respect to short-term synchronization (Negro and Farina 2011b), did not change with pain. Although it must be noted that the number of motor unit pairs analyzed constitutes a small sample with respect to all active motor units, these results indicate that the correlation in synaptic inputs for the motor units analyzed did not change during experimental muscle pain. We note that, contrary to this study, short-term synchronization was observed to increase with postexercise muscle soreness (another experimental pain model) in an arm muscle (Dartnall et al. 2008), which could be mediated by the increase in common inhibitory inputs to the motoneurons (Mattei et al. 2003; Taylor and Enoka 2004; Türker and Powers 2001). This disagreement may be due to different changes in common excitatory drive from the supraspinal regions between the two pain models. Moreover, postexercise muscle soreness also influences the fiber membrane properties, contrary to injection of hypertonic saline (Farina et al. 2004).

In conclusion, this study demonstrates that pain impairs the ability to maintain a constant force during an unaccustomed task and that this impairment is mediated by an increase in the amplitude of oscillations of the common low-frequency components of motor unit spike trains. The increase in variability in these components reflects greater variability in the synaptic input received by the motoneurons, which is due to a suboptimal compensation of changes in afferent input by the descending drive.

\section{GRANTS}

This study was partly supported by the European Research Council Advanced Grant DEMOVE (contract 267888) to D. Farina.

\section{DISCLOSURES}

No conflicts of interest, financial or otherwise, are declared by the author(s).

\section{AUTHOR CONTRIBUTIONS}

Author contributions: D. Farina, F.N., L.G., and D. Falla conception and design of research; D. Farina, F.N., and L.G. analyzed data; D. Farina, F.N., L.G., and D. Falla interpreted results of experiments; D. Farina and F.N. prepared figures; D. Farina drafted manuscript; D. Farina, F.N., L.G., and D. Falla edited and revised manuscript; D. Farina, F.N., L.G., and D. Falla approved final version of manuscript; F.N. and D. Falla performed experiments. 


\section{REFERENCES}

Bandholm T, Rasmussen L, Aagaard P, Diederichsen L, Jensen BR. Effects of experimental muscle pain on shoulder-abduction force steadiness and muscle activity in healthy subjects. Eur J Appl Physiol 102: 643-650, 2008

Berg RW, Alaburda A, Hounsgaard J. Balanced inhibition and excitation drive spike activity in spinal half-centers. Science 315: 390-393, 2007

Christou EA, Jakobi JM, Critchlow A, Fleshner M, Enoka RM. The 1- to $2-\mathrm{Hz}$ oscillations in muscle force are exacerbated by stress, especially in older adults. J Appl Physiol 97: 225-235, 2004.

Dartnall TJ, Nordstrom MA, Semmler JG. Motor unit synchronization is increased in biceps brachii after exercise-induced damage to elbow flexor muscles. J Neurophysiol 99: 1008-1019, 2008.

Davey NJ, Ellaway PH, Stein RB. Statistical limits for detecting change in the cumulative sum derivative of the peristimulus time histogram. $J \mathrm{Neu}$ rosci Methods 17: 153-166, 1986.

De Luca CJ, LeFever RS, McCue MP, Xenakis AP. Control scheme governing concurrently active human motor units during voluntary contractions. J Physiol 329: 129-142, 1982.

Dideriksen JL, Falla D, Baekgaard M, Mogensen ML, Steimle KL, Farina D. Comparison between the degree of motor unit short-term synchronization and recurrence quantification analysis of the surface EMG in two human muscles. Clin Neurophysiol 120: 2086-2092, 2009.

Ellaway PH. Cumulative sum technique and its application to the analysis of peristimulus time histograms. Electroencephalogr Clin Neurophysiol 45: 302-304, 1978.

Enoka RM, Christou EA, Hunter SK, Kornatz KW, Semmler JG, Taylor AM, Tracy BL. Mechanisms that contribute to differences in motor performance between young and old adults. J Electromyogr Kinesiol 13: 1-12, 2003

Farina D, Arendt-Nielsen L, Merletti R, Graven-Nielsen T. Effect of experimental muscle pain on motor unit firing rate and conduction velocity. J Neurophysiol 91: 1250-1259, 2004.

Farina D, Arendt-Nielsen L, Graven-Nielsen T. Experimental muscle pain reduces initial motor unit discharge rates during sustained submaximal contractions. J Appl Physiol 98: 999-1005, 2005.

Farina D, Arendt-Nielsen L, Roatta S, Graven-Nielsen T. The pain-induced decrease in low-threshold motor unit discharge rate is not associated with the amount of increase in spike-triggered average torque. Clin Neurophysiol 119: 43-51, 2008.

Gandevia SC. Spinal and supraspinal factors in human muscle fatigue. Physiol Rev 81: 1725-1789, 2001.

Graven-Nielsen T, Arendt-Nielsen L, Svensson P, Jensen TS. Quantification of local and referred muscle pain in humans after sequential i.m. injections of hypertonic saline. Pain 69: 111-117, 1997a.

Graven-Nielsen T, Svensson P, Arendt-Nielsen L. Effects of experimental muscle pain on muscle activity and co-ordination during static and dynamic motor function. Electroencephalogr Clin Neurophysiol 105: 156-164, 1997b.

Graven-Nielsen T, Lund H, Arendt-Nielsen L, Danneskiold-Samsøe B, Bliddal H. Inhibition of maximal voluntary contraction force by experimental muscle pain: a centrally mediated mechanism. Muscle Nerve 26: 708 $712,2002$.
Hodges PW, Tucker K. Moving differently in pain: a new theory to explain the adaptation to pain. Pain 152: S90-S98, 2011.

Hodges PW, Ervilha UF, Graven-Nielsen T. Changes in motor unit firing rate in synergist muscles cannot explain the maintenance of force during constant force painful contractions. J Pain 9: 1169-1174, 2008.

Marmon AR, Pascoe MA, Schwartz RS, Enoka RM. Associations among strength, steadiness, and hand function across the adult life span. Med Sci Sports Exerc 43: 560-567, 2011.

Mattei B, Schmied A, Mazzocchio R, Decchi B, Rossi A, Vedel JP. Pharmacologically induced enhancement of recurrent inhibition in humans: effects on motoneurone discharge patterns. $J$ Physiol 548: 615-629, 2003.

Matthews PB. Relationship of firing intervals of human motor units to the trajectory of post-spike after-hyperpolarization and synaptic noise. J Physiol 492: 597-628, 1996

McGill KC, Lateva ZC, Johanson ME. Validation of a computer-aided EMG decomposition method. Conf Proc IEEE Eng Med Biol Soc 7: 4744-4747, 2004.

McGill KC, Lateva ZC, Marateb HR. EMGLAB: an interactive EMG decomposition program. J Neurosci Methods 149: 121-133, 2005.

Muceli S, Farina D, Kirkesola G, Katch F, Falla D. Reduced force steadiness in women with neck pain and the effect of short term vibration. J Electromyogr Kinesiol 21: 283-290, 2011.

Negro F, Holobar A, Farina D. Fluctuations in isometric muscle force can be described by one linear projection of low-frequency components of motor unit discharge rates. J Physiol 587: 5925-5938, 2009.

Negro F, Farina D. Linear transmission of cortical oscillations to the neural drive to muscles is mediated by common projections to populations of motoneurons in humans. J Physiol 589: 629-637, 2011a.

Negro F, Farina D. Measures of correlation between motor unit spike trains in humans (Abstract). Meeting of the German Neuroscience Society, Göttingen. T21-10B, 2011b (https://www.nwg-goettingen.de/2011/upload/file/ Proceedings_2011.pdf).

Nordstrom MA, Fuglevand AJ, Enoka RM. Estimating the strength of common input to human motoneurons from the cross-correlogram. J Physiol 453: 547-574, 1992.

Semmler JG, Nordstrom MA, Wallace CJ. Relationship between motor unit short-term synchronization and common drive in human first dorsal interosseous muscle. Brain Res 767: 314-320, 1997.

Semmler JG, Nordstrom MA. A comparison of cross-correlation and surface EMG techniques used to quantify motor unit synchronization in humans. $J$ Neurosci Methods 90: 47-55, 1999.

Smith R, Pearce SL, Miles TS. Experimental muscle pain does not affect fine motor control of the human hand. Exp Brain Res 174: 397-402, 2006.

Taylor AM, Enoka RM. Optimization of input patterns and neuronal properties to evoke motor neuron synchronization. J Comput Neurosci 16: 139-157, 2004.

Tucker K, Butler J, Graven-Nielsen T, Riek S, Hodges P. Motor unit recruitment strategies are altered during deep-tissue pain. J Neurosci 29: 10820-10826, 2009.

Türker KS, Powers RK. Effects of common excitatory and inhibitory inputs on motoneuron synchronization. J Neurophysiol 86: 2807-2822, 2001.

Yao W, Fuglevand RJ, Enoka RM. Motor-unit synchronization increases EMG amplitude and decreases force steadiness of simulated contractions. $J$ Neurophysiol 83: 441-452, 2000. 